\title{
La négation verbale en mokša dans une perspective comparative
}

Mokša Mordvin verbal negation in a comparative perspective

\section{Arnaud Fournet}

\section{(2) OpenEdition}

\section{Journals}

Édition électronique

URL : https://journals.openedition.org/efo/142

DOI : $10.4000 /$ efo. 142

ISSN : 2275-1947

Éditeur

INALCO

\section{Édition imprimée}

Date de publication : 1 janvier 2011

ISBN : 978-2-296-96641-3

ISSN : 0071-2051

\section{Référence électronique}

Arnaud Fournet, «La négation verbale en mokša dans une perspective comparative », Études finnoougriennes [En ligne], 43 | 2011, mis en ligne le 14 février 2014, consulté le 08 juillet 2021. URL : http:// journals.openedition.org/efo/142; DOI : https://doi.org/10.4000/efo.142

Ce document a été généré automatiquement le 8 juillet 2021.

\section{(c) (7) (9)}

Études finno-ougriennes est mis à disposition selon les termes de la Licence Creative Commons Attribution - Pas d'Utilisation Commerciale 4.0 International. 


\title{
La négation verbale en mokša dans une perspective comparative
}

Mokša Mordvin verbal negation in a comparative perspective

\author{
Arnaud Fournet
}

1 L'article s'intéresse à différentes formes servant à l'expression de la négation verbale en mokša. Il apparaît que cette langue possède une forme spécifique pour presque chaque temps. Cette particularité peut d'ailleurs être utilisée pour identifier les différents modes et temps du verbe en mokša. après avoir décrit les différentes formes du mokša et de l'autre langue mordve, l'erzya, l'article entreprend une comparaison avec d'autres langues de l'Eurasie, qu'elles soient ouraliennes ou altaïques.

\section{Introduction}

2 En général, la négation verbale dans les langues européennes se fait à l'aide d'une unité dont le signifiant est stable : en français ne ... pas, en allemand nicht, en italien non, en basque $a z$, etc. Le basque, bien qu'il ne soit pas une langue indo-européenne, n'est pas une exception à cette tendance aréale. En français, la situation est un peu plus complexe puisque la charge négative de ne, héritée du latin, a été progressivement transférée à d'autres mots : pas, point, mie, goutte, qui étaient à l'origine des noms. Mais en français moderne, dans la langue parlée tout spécialement, la situation pratique est que la charge négative est entièrement portée par le segment pas. Les autres formes sont obsolètes ou un peu dialectales et ne est en outre fréquemment omis dans la langue informelle. L'allemand présente des variantes non standard telles que nee. En anglais, not possède une variante atone n't, mais le point en discussion ici est que la négation verbale trouve une expression segmentale unique, qui est insensible aux verbes ou aux temps et modes niés. On pourrait être tenté ou fondé de croire que cette situation en apparence économique et « logique » serait fréquente voire existerait dans la plupart des langues. C'est un fait universel que toutes les langues permettent l'expression de l'absence ou de la négation, mais il n'est pas universel que cette faculté se réalise par le truchement d'une unité unique et stable. Certaines langues, comme le 
chinois et ses centaines de millions de locuteurs, ont de fait plusieurs segments pour exprimer la négation verbale. Un exemple plus modeste en nombre de locuteurs est la langue mokša. Son examen est tout à fait intéressant et stimulant. Car dans cette langue ouralienne parlée en Russie, le signifiant de la négation verbale est variable suivant les différents modes verbaux. D'ailleurs à l'inverse, ces variations peuvent contribuer à déterminer les modes du verbe en mokša, que l'article propose de décrire en utilisant aussi ce critère. Notre approche permet de poser un regard distancié sur les descriptions disponibles de cette langue, qui ne vont pas sans quelques difficultés sur certains points.

\section{Le mode indicatif : La négation /af/ aф}

3 Le mode indicatif comprend quatre temps : trois simples et un quatrième auxilié. Les trois temps simples sont le présent, l'imparfait et le prétérit, auxquels s'ajoute le futur. La négation de l'indicatif, à tous les temps sauf le Prétérit que l'on examinera plus loin, prend la forme /af/ <aథ> qui se place normalement devant le verbe. Nous illustrons les paradigmes avec le verbe /jaka/ <яка> « marcher » :

Mokša /jakan/ <якан> « je marche » => /af jakan/ <аФ Якан>

Le futur se construit avec le verbe /karma/ <карма> " commencer» au Présent avec une des formes dites d'infinitif /jakama/: /karma-n jakama/ <карман якама> « је marcherai » => /af karma-n jakama/ <аф карман якама>.

5 L'imparfait est plus complexe et il existe deux façons de former la négation de ce temps : (1) /jakalen/ <якалень $>^{1}$ « je marchais » => /af jakalen/ <аф якалень> « je ne marchais pas» ou bien (2) /jakalen/ <якалень> «je marchais» => /afəlen jaka/ <афолень яка> « je ne marchais pas ». Les suffixes de l'imparfait /(ә)l/ et de P1 /(e)n/ peuvent être suffixés soit au verbe /jaka/ soit à la négation /af/. En pratique, il semble que la deuxième possibilité soit d'ailleurs statistiquement la plus fréquente.

Dans Aliamkin (2000, p. 127) et Zaicz (1998, p. 198) les temps présent, imparfait et prétérit sont appelés respectivement: présent (тяниень пингсь), premier passé (васенце ётай пингсь) et second passé (омБоце ётай пингсь). Zaicz (1998) est en fait surtout une description de l'erzya, bien que le titre en soit « Mordva ». En dépit de cette limitation intrinsèque, nombre de traits décrits en erzya sont souvent acceptables pour le mokša, même s'ils ne peuvent pas être directement déduits de l'article de Zaicz. On peut aussi noter qu'Ahlquist (1861) ne mentionne pas l'existence de l'imparfait, ce qui malheureusement tend à montrer que cette description pionnière du mokša n'est pas aussi fiable qu'on aimerait qu'elle fût. Zaicz $(1998$, p. 200) reprend aussi cette assertion inexacte: "The second past tense, which is lacking in Moksha, refers to events in the past, which either lasted long or habitually recurred ${ }^{2}$.»

7 Comme dans beaucoup de langues, le présent peut exprimer les actions futures, comme l'indique déjà Ahlquist (1861, p. 45): « das Präsens [vertritt] in dieser Mundart auch das Futurum $^{3}$ » ou encore Zaicz (1998, p. 199): « The present tense could more properly be termed a non past, as it often refers to the future. $»^{4}$ Cet emploi du présent est appelé futur simple (простой сай пингсь) par Aliamkin (2000, p. 128). Le futur périphrastique est quant à lui appelé futur composé (сложнай сай пингсь). Dans les faits il n'est pas certain que le futur composé soit vraiment une composante native du mokša, mais il faut peut-être y voir le calque du futur russe Быть + Verbe. De fait la même construction 
périphrastique avec d'autres verbes peut aussi avoir un sens futur : par exemple arsems "penser, envisager », ce qui rappelle incidemment le cas de l'anglais will. Les différents suffixes existant à l'indicatif peuvent être présentés ainsi :

Tableau des suffixes pronominaux de l'indicatif

\begin{tabular}{|l|l|l|l|l|l|l|}
\hline & P1 & P2 & P3 & P4 & P5 & P6 \\
\hline Présent & $-n$ & $-t$ & $-j$ & $-t-a m a$ & $-t-a d a$ & $-c ̧ t<*^{*} h t$ \\
\hline Imparfait & $-l e-n$ & $-l e-t$ & $-s$ & $-l-e m e$ & $-l-e d e$ & $-(j) s t$ \\
\hline Prétérit & $-(j) n$ & $-(j) t$ & $-s$ & $-(e) m e$ & $-(e) d e$ & $-(j) s t$ \\
\hline
\end{tabular}

8 À noter que (1) le mokša n'a quasiment pas d'harmonie vocalique et par conséquent les désinences sont sensiblement les mêmes quelle que soit la base verbale suffixée, (2) les suffixes sont les mêmes à la forme négative et affirmative.

9 L'ensemble des formes est le suivant au présent: (/af/) /jakan/, /jakat/, /jakaj/, / jakatama/, /jakatada/, /jakaçt/5. À l'imparfait, existent deux séries de formes : (/af/) / jakalen/, /jakalet/, /jakaleme/, /jakalede/ ou bien /afəlen/, /afəlet/, /afəleme/, / afəlede/ suivies de /jaka/, suivant que les désinences pronominales sont affixés à la racine verbale ou à la négation elle-même.

On peut conclure la description de <aథ> [af] en indiquant que ce morphème est une des très rares unités du mokša qui se comporte comme un préfixe : <афсатыкс> [afsatiks] «insuffisance», dérivé de la racine <caT-> [sat] "suffire, être assez». Bien que typologiquement agglutinante la langue mokša n'ignore pas entièrement la notion de préfixe.

\section{Le mode indicatif : le cas du prétérit}

11 Comme indiqué plus haut en début d'article la négation du prétérit est différente de celle des autres temps : elle a deux formes /af/ аш, qui est courant, et /iz/, /іz/ изь, qui est plus littéraire. Les formes P3 et P6 étant syncrétiques entre l'imparfait et le prétérit, elles recourent à ces deux morphèmes:/jakas/ <якась> «il, elle marchait, a marché » => /a Эəz jaka/ <ашезь яка>. La forme **/afs jaka/ est impossible.

L'ensemble des formes est le suivant au prétérit: à la forme affirmative /jakajn/, / jakat/, /jakas/, /jakame/, /jakade/, /jaka(j)st/, à la forme négative avec aш : /a〕ən/, /

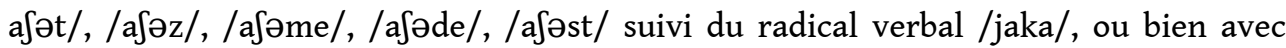
изь : /izen/, /izet/, /iz/, /izeme/, /izede/, /ist/ suivi du radical verbal/jaka/. On peut noter que la forme P3Sg /iz/ reste nue, alors que P3Pl reçoit le suffixe $-t$ du pluriel nominal.

Certains auteurs mentionnent d'autres possibilités pour l'expression de la négation. Ahlquist (1861, pp. 50-51), la première description dédiée au mokša spécifiquement, indique des formes telles que ašin jaka « je ne marche pas » reposant sur /aš/ <aW» « il n'y a pas » au Présent. Un problème est que cette formulation ne peut pas être trouvée dans les manuels ou les dictionnaires contemporains du mokša. Pour cette raison, elle 
semble douteuse, même si on peut aussi envisager qu'elle était un trait dialectal obsolescent de la variété de mokša septentrional étudiée par Ahlquist au milieu du $\mathrm{XIX}^{\mathrm{e}}$ siècle. Il est probable qu'elle soit tout simplement erronée. Paasonen (1903, p. 22) cite une forme mokša : <ašən » p'el ${ }^{\mathrm{L} t}$ » « «e n'avais pas peur ». Aliamkin (2000, p. 148) indique en effet que /aS/ peut être utilisé comme synonyme de /iz/ pour les formes verbales négatives au Passé, mais il ne cite que des exemples dans la voix médiopotentielle. Rédei $(1988$, p. 68) qui liste tous les segments à valeur négative attestés dans les langues ouraliennes mentionne /aš-/, mais pour le Passé seulement. L'autre possibilité /iz/ ou /iz/ <Изь>, est écrite <ez> par Ahlquist (1868:50-51) ou <ez, әz> par Paasonen (1903, p. 22). Car aucun de ces auteurs n'a une notation stable et cohérente du phone [i]. Elle est décrite dans Aliamkin $(2000$, p. 148) et le dictionnaire de Serebrennikov (1998, p. 202) en fournit un exemple: /izen jaka/ <изень яка> 'я не ходил'. Un autre dictionnaire (plus petit) : Herrala-Feoktistov (1998) ne liste pas cette unité.

Les équivalents erzya des phrases ci-dessus sont (Zaicz 1998, p. 198): (1) Erzya: / kundan/ <кундан> "j'attrape»=> /a kundan/ <а кундан>, (2) Erzya: /kundin/ <кундинь> «j'ai attrapé » => /ez-in kunda/ <эзинь кунда>. L'erzya a encore un segment négatif court /a/ pour lequel il n'y a pas d'équivalent en mokša moderne. Dans Witsen (1692) on peut trouver un exemple de cette négation courte /a/:<amidu> glossé en néerlandais <leeft> "vivant ». Cette forme peut être analysée comme un participe négatif: "non-mort» = «a-mid-u». Un autre exemple est <ayrista> glossé <zyn nuchteren> "être sobre ", qui correspond sans doute à <a-ired-sta> "non-ivre ». Les Mordvinen [Mordves] de Witsen (1692) sont bien connus pour être des Mokšas. Il semble que le mokša ait perdu la forme /a/ à une époque assez récente et ne possède plus que la forme longue /af/. D'après /apak/ /af/ doit probablement être analysé comme un composé de /a/ et /p(pa)/.

15 Il convient de noter que la suffixation des pronoms sur le segment négatif au lieu du verbe existe aussi en finnois : kävele-n 'je marche » => e-n kävele " je marche », mais pas en hongrois: gyalogolom $=>$ nem gyalogolom. Ce phénomène est souvent décrit comme étant un « verbe négatif ». Le mokša va plus loin que le finnois puisque le Passé ne peut pas être suffixé au segment négatif en finnois, contrairement à ce qui est possible en mokša : finnois ot-a-n 'je prends »=> e-n ota and ot-i-n 'j'ai pris »=> e-n otta-nut. En finnois e-n 'je ne ... pas » est indifférent à la catégorie du temps (et du mode). On peut noter que les dictionnaires de langue mokša inventorient /af/ comme étant une particule (частица), et non un verbe. À mon avis, ce morphème de négation en finnois ne doit pas être considéré comme un verbe. D'un point de vue strictement formel, les suffixes pronominaux sont transférés du verbe au segment négatif $e^{-}$, mais cette contingence formelle n'en fait pas un verbe. Notons que Zaicz (1998, p. 199) considère cette particule comme étant un verbe : «this particle probably descends from the base of the pFU negative verb *e- $*^{* a ̈-}$ (with metaphony *e/ä $>*_{a}$ in $*_{e}$-wole $>$ avolj 'isn't') $)^{6}$. Par ailleurs Erzya avol « n'est pas » ne doit pas être confondu avec mokša afəl

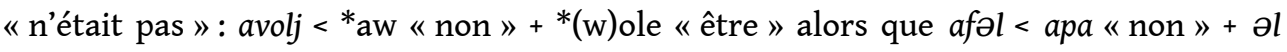
'Imparfait ». Rédei (1988:69) ne postule pas de verbe négatif en proto-ouralien : « [P]U $*_{e}$ war ursprünglich - weil einsilbig - sicher kein Begriffswort, d. h. kein Verb für 'nicht sein', sondern eine Verneinungspartikel, die später zu einem Verb wurde, d. h. sie nahm die Endungen des Grundsverbs auf : Ableitungssuffixe (vgl. elä, älä), Zeichen und 
Konjugations-endungen ${ }^{7}$.» Mais le fait que ce "mot» a une seule syllabe n'est pas en soi une raison concluante de penser qu'il ne s'agisse pas d'un verbe.

\section{Les modes imperatif et optatif}

16

La négation à l'impératif n'est pas /af/ <aథ>, mais /tja/ <TЯ> : (1) mokša /jakak/ <якаК> « marche!' => /tjat jaka/ <Тят Яка> « ne marche pas », /tjak/ existe également, (2) / jakada/ <якада> "marchez!" => / tjada jaka/ <тяда яка> " ne marchez pas ». Cette négation est celle de l'optatif, exprimé par -za- : / ulezan/ <улезан> « Puissé-je être !' => /tjazan ule/ «Puissé-je ne pas être !'. La proximité sémantique entre l'impératif, qui exprime l'ordre ou l'interdiction, et l'optatif qui exprime le souhait ou la crainte, explique sans doute que la même négation est utilisée pour les deux modes. On peut noter que les segments - $z a$ - de l'optatif et - $n$ P1sg sont suffixés au segment négatif tja.

Les auteurs diffèrent entre eux quant aux formes existant à l'optatif. Certains décrivent un paradigme défectif, par exemple Aliamkin (2000, p. 135), d'autres un paradigme complet, comme Ahlquist (1861, p. 53). En théorie, l'ensemble maxi, résultant de la combinatoire suffixale possible, est le suivant: /ulezan/, /ulezat/, /uleza/, /ulezama/, /ulezada/, /ulest/ et le paradigme négatif est alors:/tjazan/, /tjazat/, /tjaza/, / tjazama/, /tjazada/, /tjast/ suivis de /ule/. L'Impératif et l'Optatif n'ont que le temps Présent.

Il n'est pas rare en ouralien que l'indicatif et l'impératif diffèrent à la forme négative :

- finnois mene-t « tu vas » => e-t mene « tu ne vas pas », mais älä mene! « ne vas pas!»

• hongrois mész « tu vas » => nem mész « tu ne vas pas », mais ne menj! « ne vas pas!»

- vogoul Sosva śalte- $n$ « tu entres » => at śalte-n « tu n'entres pas », mais ul śalte-n! «n'entre pas!»

Il semble logique de comparer mokša tja et Vogoul at, car la perte d'une voyelle initiale n'est pas rare en mokša : /id/ " enfant »> /(i)djaka/ " poupée ». Cf. Paasonen (1903:10) and Serebrennikov (1998:171, 200). Mais le mokša n'a pas de trace de l'autre segment négatif représenté par le finnois älä et le vogoul ul. L'autre langue mordve la possède encore : erzya il'a mora «ne chante pas!' (Zaicz 1998, p. 202). Cette forme il'a est aussi utilisée pour l'Optatif en erzya: mora-za-n 'que je chante» > il'a-n mora «que je ne chante pas » (Zaicz 1998, p. 201). On est tenté de conclure que le proto-mordve a hérité les deux segments négatifs *ilja (Impératif) et *(a)tja (Optatif) et que chaque langue mordve a généralisé de façon divergente une des formes pour les deux modes.

\section{Le mode subjonctif}

Le mode subjonctif (Сослагательнай dans Aliamkin 2000, p. 132) est presque semblable à l'Imparfait de l'Indicatif, sauf pour P3 et P6 : /jakalen/, /jakalet/, /jakal/, / jakaleme/, /jakalede/, /jakałt/. Le risque de confusion explique sans doute que l'Imparfait de P3 et P6 ne soit pas /jakal/ et /jakadt/, mais soit syncrétique du Prétérit. La négation du subjonctif est exprimée par /af/ $\langle\mathrm{a} \Phi>$ avec suffixation des pronoms sur la négation: /afəlen/, /afəlet/, /afəl/, /afəleme/, /afəlede/, /afəłt//jaka/. sur le plan sémantique, ce mode décrit des actions non réalisées: possibles, impossibles, désirées ou futures. Il ne correspond pas toujours au Subjonctif du français. Par exemple, /kəda tijəl/ « comment faire? comment fera-t-on? » Ce mode est donc d'un 
emploi moins restreint qu'en français et peut s'employer seul comme prédicat principal ou unique. On le rencontre fréquemment après différentes conjonctions, en particulier /Stobə/ "pour que, afin que». En outre, le subjonctif couvre les emplois du Conditionnel : /jakalen/ signifie également «j'irais ». Dans ce sens, il peut être renforcé par /bə/ <БЫ, Ба> emprunté au russe, ce qui supprime toute ambiguïté avec l'Imparfait. Cf. Zaicz (1998, p. 200) pour des constructions similaires en erzya.

Les descriptions traditionnelles, comme Aliamkin (2000, p.137) ou Abondolo (1998, p. 201), mentionnent un mode désidératif, exprimé par le suffixe /-leksəl-/ <лексоль>. Par exemple, /jakaleksəlen/ «j'aimerais marcher ». Notre analyse est qu'il s'agit d'une unité lexicale particulière. Sur le plan formel, cette unité se suffixe au verbe, mais cela n'en fait pas un "mode » verbal pour autant. Le véritable mode de cette forme est en réalité le Subjonctif exprimé par /əl/. La négation s'exprime de façon prévisible avec / af/, négation du subjonctif : /afəleksəlen/, /afəleksəlet/, /afəleksəl/, /afəleksəleme/, /afəleksəlede/, /afəleksəłt//jaka/. L'équivalent erzya est /iksel/

Un autre mode supposé exister en mokša est le conditionnel. Ici encore, notre analyse aboutit à rejeter ce "mode» qui est en fait une conjonction de subordination. Les

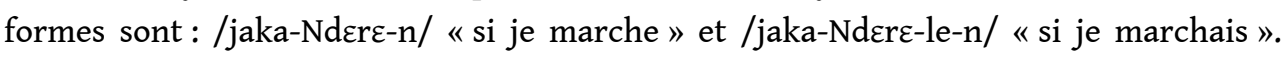

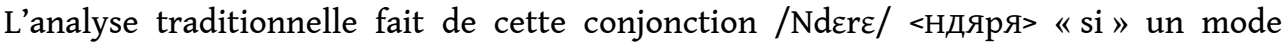
verbal d'après le critère formel qu'elle se suffixe au verbe. Cette analyse est tout à fait

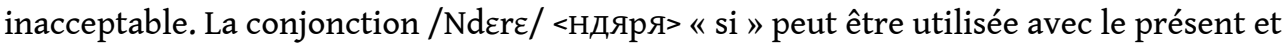

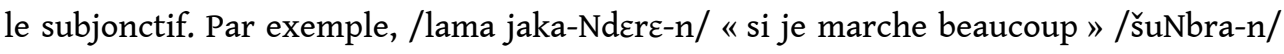
« je suis en bonne santé ». Un exemple avec le subjonctif, /lama jaka-Ndere-le-n/ « si je marchais beaucoup » /šuNbra-le-n (bə)/ « je serais en bonne santé ». La conjonction est insérée entre le verbe /jaka/ et les désinences personnelles du présent et du subjonctif : /jakaNderen/, /jakaNderet/, /jakaNderej/, /jakaNderetama/, /jakaNderetada/, /

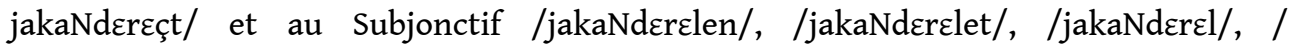

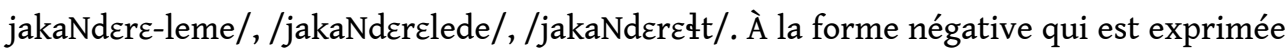
par /af/, la conjonction reste suffixée au verbe : au Présent, /af//jakaNderen/, etc. et

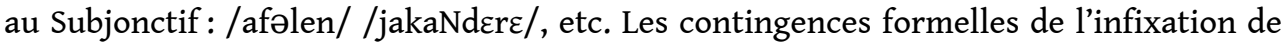
cette conjonction entre la base verbale et les suffixes temporels et pronominaux n'en font pas un mode pour autant.

Certaines unités lexicales ne semblent exister qu'au subjonctif, comme par exemple / dzmal/ <дямаль> « il ne faudrait pas, il ne faut pas », /dzmal kořta/ «il ne faudrait pas (en) parler ». Ces unités rappellent par leurs propriétés les « modals» de l'anglais. Les dictionnaires mokša-russe ne leur attribuent aucune catégorie lexicale explicite. $C f$. Serebrennikov (1998, p. 171). Une autre unité de ce type est /dعl/ <дяль> : <штоБа фкявок маци дяль кунда> « afin que /štobə/ aucune /fkevək/ oie /matsi/ ne soit / $\mathrm{d} \varepsilon \mathrm{l} /$ capturée /kunda/» (Aliamkin 2000, p. 133). Les initiales voisées en mokša signalent des emprunts. En l'occurence l'alternance morphologique entre /dzmal/ et / $\mathrm{d} \varepsilon \mathrm{l} /$ semble turkique : Cf. turcique *-ma- « not».

\section{Le cas du médio-potentiel}

Dans les descriptions traditionnelles, comme Zaicz (1998, p. 203) ou Serebrennikov (1998), la forme médio-potentielle est traitée comme un simple dérivé lexical du verbe et non comme un mode relevant de la description grammaticale du mokša. Ce point de 
vue nécessite sans doute quelques réserves. S'il s'agissait d'un simple dérivé verbal, on comprend mal pourquoi il se distingue du verbe non-dérivé à la forme négative. Si on prend comme exemple le verbe /Sařkəd/ <шархкод> " comprendre », on peut dire en mokša : /Sařkəd-i/ <шархкоди> «il comprend » => /Sařkəd-əv-i/ <шархкодови> «il peut comprendre ».

À première vue, /Sařkəd-əv/ est comparable au simple /šařkəd/ et se suffixe de la même façon. Néanmoins, à la forme négative, une dissymétrie apparaitt : (1) /af Jařkəd-

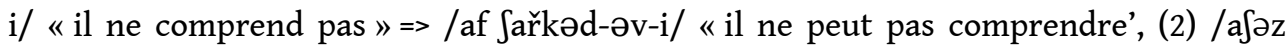
Sařkəd/ « il n’a pas compris » => /iz Jařkəd-əv-s/ « il n'a pas pu comprendre ».

$\mathrm{Au}$ prétérit, la négation n'est pas /aS/, mais /iz/, /iz/ <из> (Aliamkin 2000, p. 148). Traiter /Sařkəd-əv/ comme un item lexical ordinaire du même type que /Sařkəd/ ne permet pas de rendre compte de cette différence. Il faut noter que cette négation /iz/ est utilisée avec certains verbes dont le sens inclut la notion de possibilité : /iz kener/ «il n'a pas eu le temps, il n'a pas pu être à l'heure », /iz kener pajezdti/ «il a raté le train » (litt. « il n'a pas pu [iz] être à l'heure [kener] pour [ti] le train [pajezd] »).

En parallèle de ces formes, de sens uniquement potentiel, on en rencontre d'autres dont le sens est médio-passif et impersonnel. Par exemple : /sjormasa kořtavs/ « dans la lettre il était dit ». Ici, le verbe est « dire, parler »/kořta/ <Корхта> comme dans / mokšəks kořtan/ " je parle moksha », ou encore /af kořtavi/ « ce n'est pas dit, cela n'a pas (encore) été dit », de même /af tijevi/ "ce n'est pas fait, cela n'a pas (encore) été fait». Parfois, le trait de sens de la possibilité est plus net: /iz šařkədəv/ "c'était incompréhensible». Nous verrons avec les participes que ces formes de médiopotentiel s'écartent à nouveau des formes simples. L'ouralistique traditionnelle considère le sens médio-passif comme premier. Cf. Zaicz (1998, p. 203) : «=v- makes reflexives and medio-passives [...] this suffix has clear cognates in Saamic, Fennic, Mansi and Hungarian $\left(<\mathrm{pU}^{*}=\mathrm{w}\right) . »^{8}$

Lorsque le suffixe /(ə)v/ <(o)B> signifie "pouvoir, avoir la faculté de ", /šařkəd-əv/ peut être suffixé par tous les suffixes personnels des trois temps de l'Indicatif et par ceux du Subjonctif. Les sources ne précisent pas si on peut conjuguer /šařkəd-əv/ au futur : */karman šařkədəvəma/ ( ?). Il faut peut-être conclure de l'absence d'indication que la forme n'existe pas. Il ne semble pas non plus que cette forme puisse être combinée avec l'impératif ou l'optatif. À notre avis il existe un faisceau de particularités qui montre que la forme médio-potentielle est en mokša une voix, distincte de la voix active, et non un dérivé lexical.

\section{Les participes}

On doit distinguer deux types de formations, un groupe vivant et productif et un groupe résiduel, figé et non productif.

Les formations vivantes comprennent deux participes : présent et passé. Ils peuvent être à la fois prédicat ou bien se rapporter à un nom, auquel cas ils lui sont toujours préposés. Le participe présent est formellement identique au présent P3:/saj piNge/ «l'avenir » (litt. le temps [piNge] qui vient [saj]). La négation est /af/ : /af lama sodaj loman/ "un homme [loman] qui ne sait [sodaj] pas [af] grand-chose [lama]». Le participe passé est formé avec /f/ : /saf/ « venu », /sodaf/ « connu ». La négation est / apak/ <aпак> et le suffixe du verbe est -k (au lieu de -f) : /apak sodak loman/ « un 
homme inconnu ». Ces deux participes présent et passé peuvent être prédicat. /son saj/ saf/ <coH сай/саф> «il vient, il est venu ». Le participe passé n'a pas toujours un sens passif : /apak kořtak/ « sans avoir parlé ».

Tableau des formes productives de participe

\begin{tabular}{|l|l|l|l|}
\hline & Présent & Passé & Médio-potentiel \\
\hline Affirmation & soda-j & soda-f & soda-vi(ks) \\
\hline Négation & af soda-j & apak soda-k & af soda-vi-ks \\
\hline
\end{tabular}

En ce qui concerne les participes médio-potentiels, ils n'ont qu'une forme de présent à valeur passive. Par exemple, /sodavi(ks)/ ne signifie pas «qui peut savoir', mais « qui peut être connu ", le suffixe /ks/ étant optionnel. La négation est /af sodaviks/ "inconnaissable", le suffixe /ks/ étant dans ce cas obligatoire. De même, / Sařkədəvi(ks)/, /kelgəvi(ks)/ signifient "compréhensible", "aimable» et non pas "qui peut comprendre", "qui peut aimer». On peut comparer /apak tik tev/ "une chose [tev] non faite " avec /af tijeviks tev/ " une chose infaisable ». À noter que le participe médio-potentiel est formellement différent du participe simple, ce qui est à nouveau étrange si les verbes médio-potentiels sont simplement considérés comme des dérivés des verbes simples.

En outre, il existe trois autres formations : /ada/ <aдa>, /ez/ <e3b> et /jn/ <Hb>. Dans la langue actuelle, aucun de ces participes ne semble apte à être prédicat. Il est plus que probable que ces trois formations appartiennent à une couche ancienne et résiduelle du mokša. La formation en /ada/ est peu productive et comprend entre autres : /ozada/ " assis », /steada/ « debout ", /panzada/ " ouvert ». Ces items peuvent soit qualifier un nom, comme dans /panžada keNkJka/ "par [ka] la porte [keNkJ] ouverte [pan3]», ou encore être circonstanciels, comme dans /steada kořtaj/ «il parle debout». Ces participes en /ada/ ne semblent pas attestés à la forme négative. Ces participes sont considérés comme étant passés par Aliamkin (2000, p. 175) ou présents (simultaneous) par Zaicz (1998, p. 205). Quant à nous, cette formation nous semble plutôt relever du présent car la formation archaïque du passé semble être en /jn/. Par exemple, / kundajn/ " chassé, attrapé » sur la base du verbe /kunda/, /kundaf/ étant également possible. Il est possible que ces formations étaient auparavant en distribution complémentaire avec celles qui sont aujourd'hui les seules vivantes.

Tableau des formes vivantes et résiduelles de participes

\begin{tabular}{|l|l|l|}
\hline Participes & État actuel & État ancien (hypothèse) \\
\hline Présent prédicat & soda-j & *soda-j \\
\hline Présent non prédicat & soda-j / *soda-da & *soda-da \\
\hline Passé prédicat & soda-f & *soda-f < * soda-y \\
\hline Passé non prédicat & soda-f / *soda-n & *soda-n \\
\hline
\end{tabular}


Une autre formation, relativement fréquente, est suffixée par /z/ <3b : /avardəz kořtaj/ «il parle en pleurant ». Les formations en /z/ ne peuvent jamais déterminer un nom, ni être prédicat, et on peut les considérer comme des vrais gérondifs (des "adverbial converbs» en anglais). Suivant que l'action est simultanée ou non, la négation est /af/ ou /afəz/: /af pičədəz/ <аф пичедез> « sans s'inquiéter, sans être

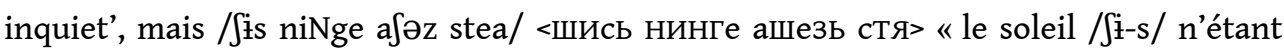
pas /ajəz/ encore /niNge/ levé /stea/ ». À noter la formation rédupliquée à valeur durative, /kftiz kfti/ «il danse /kfti/ en dansant ». Aliamkin (2000, p. 179) cite en outre une combinaison avec /tijez/ « en faisant»: /avardəz tijez/ «tout en pleurant», à valeur intensive.

\section{Récapitulatif des segments negatifs}

En résumé des descriptions et discussions qui précèdent, la langue mokša présente un ensemble très différencié de segments négatifs: $a f, i z$, aš, tja, apak, auxquels $a$ (archaïque) ou *ilja (attesté en erzya) pourraient être ajoutés.

L'approche traditionnelle de la voix médio-potentielle comme dérivé lexical ne permet pas d'établir une description complète du paradigme négatif. Il est possible que beaucoup de formes ne sont pas attestées parce qu'elles ont peu ou pas de valeur pratique en premier lieu. Presque tous les temps et modes ont ou avaient sans doute un segment spécifique :

Tableau des segments négatifs (voix active)

\begin{tabular}{|l|l|l|}
\hline & Prédicatif & Non-Prédicatif \\
\hline Indicatif présent & af $\sqrt{ }-\varnothing-[\mathrm{PP}]^{9}$ & af $\sqrt{ }-(\mathrm{e}) \mathrm{z}-\#$ \\
\hline Indicatif passé & iz/aš-[PP] $\sqrt{ }-\varnothing-\#$ & ašəz $\sqrt{ }-\varnothing-\#$ \\
\hline Indicatif imparfait & af-əl-[PP] $\sqrt{ }-\varnothing-\#$ & $($ ?) \\
\hline Subjunctif & af-əl-[PP] $\sqrt{ }-\varnothing-\#$ & $/$ \\
\hline Impératif & tja- $\varnothing-[\mathrm{PP}] \sqrt{ }-\varnothing-\#$ & $/$ \\
\hline Optatif & tja-za-[PP] $\sqrt{ }-\varnothing-\#$ & $/$ \\
\hline Participe présent & apak $\sqrt{ }-\mathrm{j}-\#$ & apak/af $($ ?) $\sqrt{ }-\mathrm{da}-\#$ \\
\hline Participe Passé & apak $\sqrt{ }-\mathrm{k}-\#$ & apak ( ?) $\sqrt{ }-\mathrm{jn}-\#$ \\
\hline
\end{tabular}

Tableau des segments négatifs (voix médio-potentielle)

\begin{tabular}{|l|l|l|}
\hline & Prédicatif & Non-Prédicatif \\
\hline Indicatif présent & af $\mathrm{V}$-əv-[PP] & $(?)$ \\
\hline
\end{tabular}




\begin{tabular}{|l|l|l|}
\hline Indicatif passé & iz/aš V-əv-[PP] & $($ ?) \\
\hline Indicatif Imparfait & $($ ?) & $/$ \\
\hline Participe présent & af V-əvi-(ks)-\# & $($ ?) \\
\hline
\end{tabular}

\section{Données comparatives ouraliennes} la négation du Passé dans plusieurs langues :

• erzya aš-, mokša aš- « il n'y a pas + passé négatif »

- mari əš- « passé négatif»

- selkup aša (Tass), assa (Turuhan), assa, ass (Ket) (présent et passé négatif)

Le segment métaphonique $e-/ i$ - avec vocalisme antérieur est répandu et utilisé pour le passé négatif (Cf. Rédei 1988, p. 69). Dans plusieurs langues, ce segment est suffixé par *-ś- :

- komi $e^{-} \sim i$ - « n'était not » (versus o- « n'est pas »)

- estonien (dial.) es, is « past » (versus ei « présent »)

- erzya ez-, mokša iz- « passé » (versus $a$ - « présent »)

Dans plusieurs langues, le segment métaphonique est utilisé pour tous les temps :

- finnois $e^{-}$, (dial.) elä; estonian $e^{-}$; same N. $i$ - or $e^{-}$

- kamasse ej- «n'était pas » (versus $e$-le- «n'est pas ») ${ }^{11}$

- nenets ńi- (mais impératif ńo- avec une voyelle postérieure), enètse ńe-12

- nganassanz ńi-nt- (avec un suffixe -nt-)

- khanty $\breve{e}$-ntə (Vah, Demianka) (avec suffixe), ă-n (Obdorsk)

La suffixation - $l$ - pour l'impératif est peut être de date finno-ougrienne :

- erzya il'a,

- mansi äl (Tavda), wil (Konda), ul (Lozva, Sosva), wul (Pelymka)

- khanty äl (V), àt (Demianka), àl (Obdorsk) (NB : -l- >-t- est un changement régulier)

On ne trouve pas d'exemple de cette forme dans la branche samoyède. En outre, le mari est it avec -t-, et non - $l$-. Rédei $(1988$, p. 68) liste kamasse el-, mais ici -l- est la marque du présent en kamasse, en opposition à -b- «passé » : nerē-l-iem «j'ai peur » nerē-b-iem «j'avais peur ». La négation de l'impératif en kamasse est $i(:)$ sans suffixe $: i$ šo $^{?}$ " [P2sg] ne viens pas !'. Ce segment $-l\left(e^{r}\right)$ - est aussi utilisé pour le Gérondif kamasse : nerēle $e^{?} \sim$ ele 
nere ${ }^{2}$ « étant effrayant $\sim$ n'étant pas effrayant ». Cette forme semble tirer son origine du morphème dérivationnel proto-ouralien *-l- à valeur fréquentative-durative.

La suffixation -p- est probablement elle aussi de date ouralienne :

- finnois epä « mé-, in- » (un préfixe) ; estonien (dial.) ep, pole, põle « non »

- erzya apa-k, mokša af, apak (Logiquement *-pp-/-Hp- >-p- car*-p- serait devenu -v-)

- komi (S) abi, (P) abu, (PO) abo-(l) « n'est pas »

- koibal abi timnem « je ne sais pas»

Il semble logique de conclure que ${ }^{*} a-p a$ était probablement une variante emphatique de la négation du Présent. Outre - $p$-, deux autres suffixations sont attestées : - $t$ - et $-k$-. Elles sont souvent utilisées pour l'impératif :

- mokša tja < *a-t-ja (optatif, impératif), Mokša, Erzya $a-p a-k$

- mari it, id- (KB) (impératif) ; ok, oyel (U) « ne ... pas » (présent)

- mansi at (Konda), at (Lozva, Sosva) (indicatif) ; äk (Tavda) (participe),

- selkoupe iki (Tass), êkka (Ket) (impératif)

Une suffixation très rare est $-n-$ :

- khanty $\breve{a}-n$ (Obdorsk) «ne ... pas»

- kamasse ene 'in-' : ene-neynōne « unschmelzbar [qui ne peut pas fondre]» (Donner $1944: 18)^{13}$

$\mathrm{Au}$ final l'inventaire comparatif de la négation verbale en ouralien peut être résumé comme suit :

Tableau des Négations verbales en ouralien

\begin{tabular}{|c|c|}
\hline s & Origines possibles \\
\hline$*_{\mathrm{a}-} / *_{\mathrm{o}-}$ & 1. Forme de base « ne ... pas» \\
\hline *a-pa- & Variante nominalisée (?) \\
\hline *ak-/*ok-; *at-/*ot-; *an-/*en- & Suffixation par déictiques \\
\hline 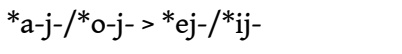 & Variante métaphonique ${ }^{*}{ }_{-j}-$ \\
\hline *al-/*o-l- ; *e-l-/*i-1- & Suffixe *-le- «fréquentatif» \\
\hline$*_{\mathrm{es}-} / *_{\mathrm{iz}}$ & Suffixation par *-ś- «P3Sg passé » \\
\hline *a-nt- ; *e-nt- & Variantes avec $-n t-$ \\
\hline *aš- & 2. « il n'y a pas » > Variante pour le passé \\
\hline
\end{tabular}

Les suffixes - $t-,-k-,-n$ - peuvent être comparés avec les déictiques proto-ouraliens ${ }^{*} t,{ }^{*} k$ and *n. Rédei $(1998$, p. 69) considère que *- $k$ - est un morphème d'impératif et que *-tserait un suffixe déverbal. Il faut néanmoins noter que - $p$ - ne peut pas être mis en regard d'un déictique proto-ouralien. Rédei (ibidem) considère qu'il s'agit d'un suffixe nominal finno-ougrien. Cette idée est de fait cohérente avec apak qui est utilisé pour des formes verbales de type participe. 


\section{Données comparatives nostratiques} "non», inam- $u$ 否む 《nier, refuser». En japonais moderne existe encore -nai «n'est pas ». Il est probable que la charge négative est portée par la voyelle initiale et non la nasale.

2. *-l- : Bomhard (2008, p. 371-372) mentionne une latérale *al-, *əl-, *el-, *ul- : « Uralic : Proto-Uralic *elä imperative of the negative auxiliary verb (Cf. Collinder 1977, p. 26). Marcantonio (2002, p. 239) describes the patterning in Finnish as follows: "A negative verbal form is used in Finnish also in the Imperative, as shown by the pair lue 'read' vs älä lue 'do=not read' (2nd Person Singular). The negative form älä is often compared with the equivalent Yukaghir el ele. Equivalent negative verbs and related isomorphic constructions are found in the majority of the Tungusic languages $(e-\sim \ddot{a}-)$, in Mongolian (e-se) (UEW 68; SSA 100) and in Dravidian ${ }^{15}$." " Bomhard (2008) tente de séparer ces deux formes, mais tout pousse à faire de ${ }^{2} a l-\left(\sim^{* ?}\right.$ əl- $)$ une variante suffixée de ${ }^{*} e$. "Rédei $\left(1988\right.$, p. 68-70) treats the negative verb ${ }^{*}$ - and the imperative *elä together, as do many others, including Collinder and Tailleur. As noted by Greenberg (2000:214), these two forms are so closely intertwined, often through suppletion, that it is difficult to distinguish one from the other. [...] Clearly, we are dealing with two 
separate forms here. The first is the Proto-Nostratic negative particle ${ }^{*} e$ 'no, not', and the second is the negative verb ${ }^{2} a l-(\sim$ *? $\partial l-)$ 'to be not so-and-so ${ }^{16}$."

Du point de vue ouralien, toute comparaison de PU *a/o-l- ou PU *e-j-l avec une autre langue fait sens seulement si -l-peut être dérivé d'un suffixe fréquentatif-duratif dans la langue en question. L'idée que les négations courtes et longues peuvent être étudiées indépendamment les unes des autres, comme le fait Bomhard (2008, p. 372), ne peut pas être acceptée. La comparaison des formes ouraliennes avec la «Proto-Altaic *ule ( -i) negative particle: Proto-Mongolian *ülü [...] > Written Mongolian ülü; Khalkha ül; Buriat üle; Kalmyk üle; Ordos üle, ülü ; Moghol la, lü, le; Dagur ul, ule; Dongxian ulie; Shira-Yughur lə; Monguor li, li.» dans Bomhard $(2008$, p. 373) a un sens parce que le mongol a une voyelle postérieure et parce qu'il ne fait pas de doute que le -l- a une valeur fréquentative-durative en mongol même : bari- « tenir, saisir » > bari-lda- « faire de la lutte »; jari- « parler » > jari-ltsa- « bavarder, converser ».

3. Initiales vocaliques: Bomhard (ibidem) inventorie plusieurs segments négatifs reposant sur ${ }^{* 2} e^{-}:$: Altaic : Proto-Altaic ${ }^{*} e$ negative particle : [1] Proto-Tungus ${ }^{*} e-$ 'not'

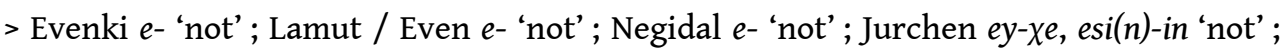
Ulch $e$ - 'not'; Orok $e$ - 'not'; Nanay / Gold $e$ - 'not'; Oroch $e$ - 'not'; Udihe $e$ - 'not'; Solon $e^{e-~ ' n o t ' . ~[2] ~ P r o t o-M o n g o l i a n ~ e-s e ~ ' n o t ' ~>~ W r i t t e n ~ M o n g o l i a n ~ e s e ~ ' n o t ' ; ~ K h a l k h a ~ e s ~ ' n o t ' ; ~}$ Buriat ehe 'not'; Kalmyk es 'not'; Ordos ese 'not'; Moghol sa, se 'not'; Dagur es 'not'; Monguor sə, sī'not ${ }^{17}$. "

Starostin-Dybo-Mudrak (2003) liste plusieurs autres segments, ne figurant pas dans Bomhard (2008) : evenk āčin, évène ān, $\bar{a} \check{c}$, neguidal āčin, mantchou littéraire aqu, oultcha ana, orok ana, nanaï anā, orotch ana, oudighe anči, solon aši. Suivant les cas, elles se laissent comparer à mordve aš-, ou bien proto-ouralien *an or *ak.

4. Plusieurs familles ont des traces d'un suffixe -j- souvent associé à des métaphonies vocaliques : ouralien $a^{-} \sim e i^{-}$, youkaghir $e l-\sim$ oj-$^{-}$, toungouse $a-q u \sim e y-\chi e$, mongol $u l-\sim e^{-}$. Le japonais présente *i-na. À voir également : mongol *-üg-əj.

\section{Conclusion}

Dans l'article, nous avons étudié les différents morphèmes utilisés en mokša pour la négation verbale et déterminé par là même les temps et modes de cette langue. De façon générale, notre étude permet de mettre au jour le système du verbe en mokša, et aussi en mordve par comparaison avec l'erzya. Sur le plan typologique, le groupe mordve offre une diversité de morphèmes négatifs exceptionnellement riche.

En outre, nous avons collecté et décortiqué le corpus de morphèmes verbaux négatifs de la famille ouralienne dans son ensemble. In fine, notre étude suggère que les différentes combinaisons à valeur négative identifiées en ouralien ont des contreparties dans d'autres langues et familles en Eurasie, offrant ainsi une triple perspective descriptive, comparative et typologique sur cette question.

L'inventaire comparatif peut être résumé dans le tableau suivant :

Tableau comparatif des segments négatifs

\begin{tabular}{|l|l|l|l|l|l|}
\hline & Ouralien & youkaghir & mongol & toungouse & japonais \\
\hline
\end{tabular}




\begin{tabular}{|c|c|c|c|c|c|}
\hline \multicolumn{6}{|c|}{ Forme de base «n'est pas, n'était pas» } \\
\hline$*_{\mathrm{a}-} / *_{\mathrm{o}-}$ & $*_{\mathrm{a}-} / *_{\mathrm{o}-}$ & $\left(c f . o^{-}\right)$ & $(c f . u-)$ & $(c f . a-)$ & / \\
\hline$-j-$ & *e(j) & oj- & $(C f . \mathrm{e}-)$ & $* \mathrm{e}-$ & $\left(c f . i^{-}\right)$ \\
\hline$-1-$ & *al-/*el- & el- / -le- & $*$ ul- & / & / \\
\hline- nt- & *ant & / & / & / & / \\
\hline \multicolumn{6}{|c|}{ Suffixation par déictiques et pronoms } \\
\hline$-\mathrm{p}-$ & $* a-(p) p a-$ & / & $\mathrm{bu}(?)$ & / & / \\
\hline$-\mathrm{k}-/^{*}-\mathrm{q}-$ & *ak-/*ok- & / & *-ugəj & *eyq- / aqu & / \\
\hline$-t-$ & *at-/*ot- & / & / & / & / \\
\hline$-n-$ & *an-/on-/en- & / & / & *an- & OJ i-na- \\
\hline *ś (P3sg) & $*_{\mathrm{es}-} / *_{\mathrm{iz}}$ & & *es- (?) & *es-in & \\
\hline \multicolumn{6}{|c|}{ « il n’y a pas» } \\
\hline *ač- & *aš- & / & *es- (?) & *ač-in & / \\
\hline
\end{tabular}

On peut noter que le groupe turcique n'a aucune trace de ces formes qu'on peut identifier en ouralien, youkaghir, mongol, toungouse et japonais. Cela peut être un argument de plus contre l'hypothèse altaïque, sans parler du coréen. En conclusion plus générale, il n'est pas exclu que notre étude puisse aussi avoir une portée génétique.

\section{BIBLIOGRAPHIE}

ABondolo Daniele M. (ed), 1998, The Uralic Languages, London and New York: Routledge.

AHLQUIST A., 1861, Versuch einer moksha-mordwinischen Grammatik nebst Texten und Wörterverzeichniss, Saint-Petersbourg: Eggers et Comp.

ALIAMKIN N.S., 2000, Mokshen Käl Morfologija, Saransk, Krasnyj Oktiabr ».

BOMHARD A. 2008. Reconstructing Proto-Nostratic, Comparative Phonology, Morphology, And Vocabulary, 2. vol. Leiden: Brill.

BUBRIH D. V., 1953, Istoricheskaja grammatika erzianskogo jazyka, Saransk: Mordovskoe knižnoe izdatel'stvo. 
BUDENZ József, 1876, Mordwinische Grammatik, Budapest.

BUDENZ József, 1899, Moksa- és erza-mordvin nyelvtan, Budapest.

COLLINDER Björn, 1955, Fenno-Ugric Vocabulary: An Etymological Dictionary of the Uralic Languages, Stockholm: Almqvist \& Viksell. (1977 Second, revised edition: Hamburg: Helmut Buske Verlag.) COLLINDER Björn (ed), 1957, Survey of the Uralic Languages, Stockholm: Almquist \& Wiksell.

COLLINDER Björn, 1960, Comparative Grammar of the Uralic Languages, Stockholm: Almquist \& Wiksell.

COLLINDER Björn (ed), 1965, An Introduction to the Uralic Languages, Berkeley: University of California Press.

DÉcsY Gyula, 1990, The Uralic Protolanguage: A Comprehensive Reconstruction, Bloomington, Indiana.

DONNER Kai 1944, Kamassisches Wörterbuch, Helsinki : Suomalais-Ugrilainen Seura.

FoURNET Arnaud, 2008, « Le vocabulaire mordve de Witsen : Une forme ancienne du dialecte zubumokša », Études finno-ougriennes, $n^{\circ} 40$, p. 57-84.

FOURNET Arnaud, 2010, Le moksha, une langue ouralienne, Saarbrücken : Éditions universitaires européennes.

FOURNET Arnaud, 2011, «Quelques éléments historiographiques concernant la famille ouralienne aux XVII et XVIII ${ }^{\mathrm{e}}$ siècles ", Beiträge zur Geschichte der Sprachwissenchaft, vol. 21.1, pp. 55-64.

FOURNET Arnaud, 2011, About the difficulty of determining the lexical classes of the Moksha language, ReVEL, vol. 9.17.

HAjDÚ Péter, 1963, Finnugor népek és nyelvek, Budapest: Gondolat kiadó.

HAJDÚ Péter, 1975, Finno-Ugrian Languages and Peoples, traduit par G. F. Cushing. London: André Deutsch.

HERRALA E. \& Feoktistov, A. 1998, Mokshalais-suomalainen sanakirja, Turku: Publications of the Department of Finnish and General linguistics of the University of Turku (48).

LAAKSO Johanna., 1992, Uralilaiset kansat, Porvoo-Helsinki-Juva.

MUNKÁCSI B., 1901, Árja és kaukazusi elemek a finn-magyar nyelvekben, Budapest.

REDÉI K. (éd.), 1986-88, Uralisches etymologisches Wörterbuch, 3 volumes, Budapest, Akadémiai Kiado.

SAmmallahti Pekka, 1988, Historical phonology of the Uralic Languages, in: Sinor, Denis (ed.) The Uralic Languages, Leiden: E. J. Brill, pp. 478-554.

Serebrennikov B. A., FeokTistov, A. P. \& Poliakova, O. E. (ed.), 1998, Mokšansko-russkij slovar ». Moscou: Izdatel'stvo Russkij Jazyk.

StAROSTIN S., DYBo A.V. \& MUDRAK O.A., 2003, Etymological Dictionary of the Altaic Language, Leiden: Brill.

VERSHININ Valerij Ivanovich 2004, 2005, 2009. Etimologičeskij slovar' mordovskih (erzjanskogo $i$ mokšanskogo) jazykov, 4 vols, Joškar-Ola: String.

WITSEN N. C., 1692, Nord en Oost Tartarye, Amsterdam: Halma.

ZAICZ G., 1998, Mordva [en fait surtout l'erzya] in: Abondolo, D. (ed.) 1998. The Uralic Languages, London and New York: Routledge. 


\section{NOTES}

1. La notation phonologique utilisée dans l'article ne note pas la palatalité induite par les voyelles antérieures /i/, /e/ et / $/ \varepsilon /$ dans les dialectes sud-orientaux du mokša: /jakalen/= [jakal'en'].

2. Le second passé, qui fait défaut en mokša, fait référence à des événements du passé qui ont duré longtemps ou bien se sont reproduit de façon habituelle.

3. Le présent exprime dans ce dialecte aussi le futur.

4. Le présent pourrait être appelé de façon plus adéquate un non-passé, car il se réfère souvent au futur.

5. Cette forme s'écrit <якайхть>/jakajht/ dans la langue littéraire. Cette orthographe étymologisante se rencontre encore de nos jours dans certaines variétés dialectales de mokša.

6. Cette particule descend probablement de la base du verbe négatif proto-ouralien e- $\sim$ *ä- (avec métaphonie *e/ä > *a dans *e-wole >> avolj 'n'est pas').

7. Proto-ouralien *e n'était originellement - car mono-syllabique - certainement pas un vrai mot, à savoir pas un verbe signifiant 'ne pas être', mais une particule de négation, qui plus tard est devenue un verbe, à savoir elle a pris les désinences des verbes : les suffixes (comparer elä, älä) et les marques de temps.

8. Le suffixe mordve $-v$ - forme des réflexifs et des médio-passifs [...] ce suffixe a des cognats clairs en saami, fennic, vogoul-mansi et hongrois (proto-ouralien ${ }^{*} w$ ).

9. $\sqrt{ }$ désigne la base verbale, -PP- les suffixes des pronoms personnels, -\# indique qu'aucun PP ne peut être suffixé à cette forme.

10. Il s'agit sans doute d'un autre segment plutôt que d'une variante suffixée. Cf. mongol -ül '(ne ...) pas' es 'il n'y (a) pas, n'était pas'.

11. En kamasse, suite à la généralisation, le suffixe *-le- de fréquentatif-duratif est devenu un marqueur du présent.

12. Le développement régulier d'initiales nasales *ń ou *y en samoyède n'a rien à voir avec une négation sur base nasale ${ }^{*}$.

13. On peut noter que cet exemple ne semble être listé nulle part : ni dans Rédei (1988) ni dans Bomhard (2008). Il est donc prudent de penser que notre inventaire n'est sans doute pas exhaustif non plus.

14. E. ouralien : Particule négative proto-ouralienne *ne > hongrois në, nëm 'ne, non'; mari nõ, ni : nó-mat, ni-ma-at, ni-mat 'rien', ni-gü 'personne'; oudmourte ni: ni-no-kin 'personne', ni-no-ku 'jamais', ni-no-mer 'rien' ; komi nõm, nem, ńem 'rien' ; mansi (Nord) nee-mäter 'rien', neem-hot ‘nulle part', neem-huunt 'jamais'; khanty (Nord) nem-hŏjat 'personne', nem-huntta 'jamais', nemətti, nəməttə 'rien'.

15. Ouralien: proto-ouralien *elä 'Impératif du verbe auxiliaire négatif' (Cf. Collinder 1977, p. 26). Marcantonio (2002, p. 239) décrit ainsi le finnois : «Une unité verbale négative est utilisée en finnois aussi à l'Impératif, comme le montre la paire lue 'lis !' vs älä lue 'ne lis pas !' ( $2^{\mathrm{e}}$ Personne Sg.). La forme négative älä est souvent comparée avec son équivalent youkaghir el ele. Des verbes négatifs similaires et des constructions isomorphiques parentes existent dans la majorité des langues toungousiques ( $e$ - $\ddot{a}$-), en mongol ( $e$-se) (UEW 68 ; SSA 100) et en dravidien. 16. Rédei (1988:68-70) traite ensemble le verbe négatif ${ }^{*} e$ - et l'impératif *elä, comme font beaucoup d'autres, dont Collinder et Tailleur. Comme l'a noté Greenberg (2000, p. 214), ces deux unités sont si intimement liées, souvent par supplétion, qu'il est difficile de les distinguer l'une de l'autre. [...] Clairement, il s'agit de deux unités distinctes. La première est la particule négative proto-nostratique ${ }^{* ?} e$ 'ne, non', et la seconde est le verbe négatif ${ }^{?} a l-\left(\sim^{* ?} \partial l-\right)$ 'n'être pas comme ceci ou cela'.

17. Altaïque : proto-altaïque ${ }^{*} e$ particule négative : [1] proto-toungouse ${ }^{*} e-$ 'ne...pas' > evenki $e$ -

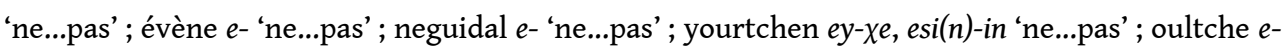


‘ne...pas' ; orok $e$ - ‘ne...pas' ; nanaï $e$ - ‘ne...pas' ; orotche $e$ - ‘ne...pas' ; oudégué $e$ - 'ne...pas' ; solon $e$ 'ne...pas'. [2] proto-mongol $e$-se 'ne...pas' > mongol littéraire ese 'ne...pas'; khalkha es 'ne...pas'; bouriate ehe 'ne...pas' ; kalmouke es 'ne...pas' ; ordos ese 'ne...pas' ; moghol sa, se 'ne...pas' ; dagour es 'ne...pas'; monguor sə, sī ‘ne...pas'.

\section{RÉSUMÉS}

Certaines langues utilisent un morphème stable et unique pour exprimer la négation verbale. D'autres en ont plusieurs suivant les modes et les temps du verbe. C'est le cas de la langue ouralienne mokša-mordve. Cette situation peut d'ailleurs être utilisée à des fins descriptives pour déterminer les modes et les temps dans cette langue et cette approche rend apparent le fait que les descriptions traditionnelles du mokša ne peuvent pas être acceptées sans quelques réserves. En particulier cela soulève la question de ce qui fait partie de la grammaire ou bien du lexique de cette langue. Dans un deuxième temps le mokša est comparé à d'autres langues du nord de l'Eurasie.

Some languages use a stable and unique segment to express verbal negation, like 'not' in English. Some others have several units according to the moods and tenses of the verb. This is the case in the Uralic Mokša-Mordvin language. This feature can be used for descriptive purposes to determine the moods and tenses of this language and this approach makes it apparent that the traditional descriptions of Mokša cannot be accepted without some reservations. In particular it raises the theoretical issue of what belongs to the lexical or grammatical component of a language. Once properly described, Mokša can be compared with other languages of northern Eurasia.

\section{INDEX}

Thèmes : linguistique

Mots-clés : négation, mokša

Keywords : negation, Linguistics, Mordvinian, Moksha

disciplines allemand, altaïque, anglais, basque, bouriate, chinois, coréen, dagour, langues dravidiennes, enets, estonien, évène, evenki, finnois, français, hongrois, langues indoeuropéennes, langues indo-iraniennes, italien, kalmouke, kamasse, khalkha, khanty, komi, latin, mari, moghol, mongol, monguor, mordve, nanaï, neguidal, nénetse, nganassan, ordos, orok, orotche, oudégué, oudmourte, oultche, proto-altaïque, proto-mongol, proto-mordve, protoouralien, proto-toungouse, samoyède, selkoupe, solon, toungouse, vogoul, yourtchen, yukaghir 